\title{
BLICKDIAGNOSE-QUIZ
}

Rötung, Schuppung, Plaques

\section{Haben Sie die Dermatose erkannt?}

\section{Auflösung von Seite 5}

- Der Allgemeinzustand des Patienten war insgesamt deutlich reduziert. Der Patient klagte über verminderte Leistungsfähigkeit, er berichtete über viele „grippale Infekte". Es bestand Alkoholabusus. Als Leiharbeiter schlief er häufig in Unterkünften mit mangelhaftem Hygienestatus, die Sexualanamnese war nicht exakt eruierbar. Sonstige Grundkrankheiten sowie die regelmäBige Einnahme von Medikamenten wurden ebenso negiert wie die Frage nach Psoriasis oder Neurodermitis innerhalb der Familie. Aufgrund des Hautbefundes sowie der Allgemeinanamnese stellten wir die Verdachtsdiagnose einer krustösen Scabies. In der Auflichtmikroskopie konnten wir die Krätzmilben (Sarcoptes scabiei) eindrucksvoll nachweisen und somit die Diagnose einer Scabies bestätigen (siehe Abb.). Initialer HIV-Test sowie die Diagnostik bezüglich STD (sexual transmitted diseases) waren unauffällig.

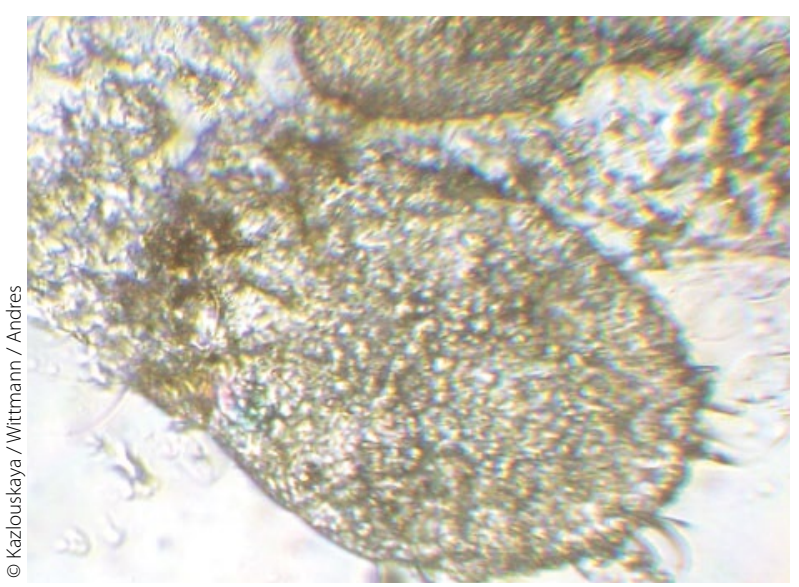

Krätzmilben (Sarcoptes scabiei) in der Auflichtmikroskopie.

Aufgrund des sehr ausgeprägten Befundes wurde der Patient hospitalisiert und antiscabiös topisch mit Permethrin Creme (5\%) für zwölf Stunden am gesamten äußeren Integument einschließlich Kopfhaut sowie systemisch mit Ivermectin 0,2 mg/kg KG per os behandelt. Dieses Schema wurde insgesamt dreimal wiederholt. Anzumerken bleibt, dass Ivermectin in Deutschland derzeit nicht zugelassen ist und daher in dieser Situation eine schriftliche Aufklärung und das Einverständnis des Patienten dringlich zu empfehlen sind. Zudem erfolgte eine begleitende abschuppende und antientzündliche Therapie.

Die Hautveränderungen heilten komplett $a b$, jedoch stellte sich drei Monate später ein Rezidiv ein. Wiederum erfolgte die identische Therapie, jedoch zeigte nun bei der zweiten Vorstellung der HIV-Test (ELISA und Western blot) ein positives Resultat.

Klinisch ist für die krustöse Variante der Scabies (Scabies norvegica) ein psoriasiformes Bild typisch, häufig auf dem Boden einer Erythrodermie. Zusätzlich imponieren an Fußsohlen und Handtellern auffällige Hyperkeratosen. Interessanterweise kann der Juckreiz bei dieser Form manchmal nicht das führende Symptom darstellen.

\section{Scabies norvegica vor allem bei Immunsupprimierten}

Die Scabies norvegica tritt vor allem bei immunsupprimierten Patienten auf, da sich die Krätzmilbe hier ungehemmt in der Haut vermehren kann. Daher sollte unbedingt der Immunstatus bei diesen Patienten abgeklärt und im Verlauf kontrolliert werden. Wie im vorliegenden Fall kann initial ein HIV-Test falsch-negativ sein, wenn die Blutentnahme in das Fenster der Serokonversion fällt. Alle Kontaktpersonen sollten sich zumindest in der Klinik vorstellen, idealerweise sollte parallel eine Behandlung erfolgen. Abschließend sei noch auf die Leitlinie "Skabies" (http://www. derma.de/fileadmin/derma/pdfs/LL_Skabies_2006_07_17.pdf) der DDG (Deutschen Dermatologischen Gesellschaft) und ADI (Arbeitsgemeinschaft Dermatologische Infektiologie) sowie die „European STD Guidelines" (http://www.iusti.org/sti-information/pdf/guidelines.pdf) hingewiesen, die wertvolle und sehr praktische Informationen beinhalten.

Keywords: Norwegian scabies - immunosuppression

- Dr. Kazlouskaya Viktoryia, MD, $\mathrm{PhD}^{1}$, Wittmann Christa, $\mathrm{MD}^{2}$, Priv.-Doz. Dr. Andres Christian, MD 1,3,4

${ }^{1}$ Ackerman Academy of Dermatopathology, New York, USA

${ }^{2}$ Lenox Hill Hospital, New York, USA

${ }^{3}$ Klinik für Dermatologie und Allergologie der Technischen Universität am Biederstein München

${ }^{4}$ Gemeinschaftspraxis für Dermatohistologie Dr. Gummer \& PD Dr. Andres, Sonnenstraße 7, 80331 München 